boundary communication, and it is useful upon any readmission as discharge summaries are more readily accessed than the paper notes.

\section{P-16 SIMPLE, ACCURATE IDENTIFICATION OF APPROPRIATE HOSPITAL INPATIENTS FOR ADVANCE CARE PLANNING BY COTE}

Jean Potter, Roberta Jordan, Catherine Pye, Yolande Saunders. The Hillingdon Hospital NHS Foundation Trust, London, UK

10.1136/bmjspcare-2018-hospiceabs.41

Background Identification of patients approaching the end of life and advance care planning (ACP) with them can improve patient outcomes and reduce hospital deaths. But this aspect of care often receives little attention in the hospital inpatient setting.

Aims (i) To estimate the proportion of a sample of COTE hospital inpatients that are identified, by their own team, as likely to be in the last $6-12 / 12$ of life; (ii) To describe these patients in terms of: likelihood of death in 6-12/12 using Gold Standards Framework Prognostic Indicator Guidance (GSF PIG); clinical complexity (using the ACE27 comorbidity score); content of any advance care plans; (iii) To ascertain the number of patients from this sample who died in the subsequent $6 / 12$.

Methods COTE Consultant led MDTs prognosticated 57 inpatients under their care during one week in March 2016, using two screening questions. All patients thought to have an at least moderate expectation of death (based on a modified Surprise Question: 'Would you be surprised if this patient were still alive in the next 6-12/12?') had their notes reviewed using a standardised proforma. Six months later the number of deaths was ascertained using hospital and community databases.

Results $70 \%$ patients were considered likely to be in the last $6-12 / 12$ of life. $98 \%$ had this prognostication confirmed with GSF PIG. 68\% patients had complex care needs, but few had advance care plans recorded. Over one third died within six months. The modified Surprise Question correctly identified patients who went on to die in the following six months with a sensitivity $61 \%$ and specificity $88 \%$.

Discussion Many COTE inpatients are in the last 6-12/12 of life. One question identifies $>60 \%$ with high specificity, and this is now an integral part of the weekly COTE MDT discussion, and will prompt ACP discussions with patients and families.

\section{P-17 ADVANCE CARE PLANNING IN DEMENTIA - TRAINING NEEDS ANALYSIS FOR EFFECTIVE FACILITATION}

Karen Harrison Dening, Caroline Scates. Dementia UK, London, UK

10.1136/bmjspcare-2018-hospiceabs.42

Advance care planning (ACP) is a voluntary process of discussion and review to help an individual who has capacity to anticipate how their condition may affect them in the future. Dementia is a progressive and life limiting illness (Sampson \& Harrison Dening, 2013), where the mental capacity to make decisions can be lost fairly early on in the illness. There is a small, growing literature on ACP and its application in dementia in the UK highlighting that its uptake is small and patchy due to several factors. People with a life-limiting illness, especially dementia, are not routinely consulted about their wishes and preferences for future care. There are several potential barriers that may contribute to this in the general population, such as, procrastination, or waiting to do it later, dependence on family for decision making. However, in dementia, there are several additional barriers to initiating ACP one of which is a reluctance on the part of professionals to undertake such difficult initial conversations. This is in part due to the perception that they lack the knowledge, skills and experience to do so (Harrison Dening, Jones \& Sampson, 2011).

Admiral Nurses (AN) are expert practitioners in the case management of families affected by dementia. A growing aspect of their role is in supporting ACP. However, as more and more are recruited Dementia UK wanted to ensure there was a consistency across the workforce in their ability to facilitate the effective initiation and development of ACP. This paper discusses a UK-wide training needs analysis of ANs undertaken during master classes on palliative and end of life care in dementia. It will discuss results and the development of an educational intervention to equip ANs in this activity.

\section{P-18 ANTICIPATORY CARE PLANNING ON AN ACUTE GASTROENTEROLOGY WARD}

Karim El-Shakankery, Daisy Riddle, Lucy Pain. Queen's Hospital, Romford, London, UK

\subsection{6/bmjspcare-2018-hospiceabs.43}

Background With a rapidly ageing population and advances in medicine, hospital inpatients are increasingly frail with complex needs, and frequently die in hospital. Advance Care Planning (ACP) is a valuable process supporting clinicians to provide patients with the right care, at the right time, in the right place.

The Gold Standards Framework (GSF) is a structured approach to ACP for patients likely to be in their last year of life. In our Trust geriatric wards have been using GSF for several years, however acute medical wards have less experience of ACP, despite many of their inpatients being in their last year of life due to both malignant and non-malignant conditions.

Aims To assess the quality and quantity of ACP on a Gastroenterology ward at Queen's Hospital, Romford before and after implementation of GSF. We also aimed to explore:

- The proportion of inpatients dying within one year

- Significant diagnoses

- Impact of ACP on length of stay, number of readmissions and place of death.

Methods Retrospective review: We identified all admissions to the ward over June 2017. All patients dying within one year were selected; data was extracted from medical notes. Data included diagnosis, length of stay, number of subsequent admissions, preferred place of care, DNAR status, and use of any formal ACP documentation.

Prospective audit: In June 2018 we implemented GSF to the ward. All patients identified were offered ACP. Comparable data was collected prospectively.

Results In June 2017, 106 patients were admitted to the ward; of these 27 have died to date. We continue to analyse 
these records. We are currently collecting prospective data throughout June 2018.

Conclusion Our data supports existing literature indicating that approximately $1 / 3$ of inpatients are in their last year of life (Clark, Armstrong, Allan et al., 2014). Full results will be presented at the 2018 Hospice UK Conference.

\section{P-19 AVOIDING THE 'HALLWAY HUDDLE' ... PROMOTING ADVANCE CARE PLANNING}

Jane Garner. Mountbatten, Isle of Wight, UK

\subsection{6/bmjspcare-2018-hospiceabs.44}

Rationale/background Advance care planning (ACP) is recognised as being key to improving end of life care. A high proportion of people feel comfortable talking about death and dying but historically conversations have been left until a palliative diagnosis or not done at all. Families are often left to guess and make difficult decisions about care and treatment when a loved one loses capacity.

Objective Aims of the project include the development of tools, a training programme and resources to help transform professional and public attitudes to ACP and reduce the barriers, including a lack of resources, limited public awareness and professional hesitancy.

Method An assessment of local recording systems led to the development of a new template to simplify the creation of ACPs on the electronic patient record system. A public ACP booklet has been produced for anyone to record their wishes and preferences and to raise the profile of ACP. Training has commenced at the hospice, in care homes and at other locations, to improve competence.

Results The electronic template is operational and has also been adopted by GPs and district nurses. The hospice is leading by example, aiming to offer $100 \%$ of appropriate patients an ACP conversation. The new ACP booklet is available to download online or pick up from various outlets. 200 people have attended ACP training at the hospice: evaluations demonstrate an improvement in knowledge and confidence.

Conclusion It is likely that the role of hospice staff will change from creating ACPs to reviewing existing documents when patients are referred. There is no 'right time' to start an ACP, but whether in good health or not, nobody is immune from sudden illness or accidents; an ACP can help ensure everyone receives care and treatment in line with their wishes and preferences.

\section{P-20 ADVANCE CARE PLANNING - WHAT ARE WE PROMISING PATIENTS?}

${ }^{1}$ Phil Moore, ${ }^{2}$ Matthew Doré, ${ }^{2}$ Derek Willis. ${ }^{1}$ Keele University, Stoke, Staffordshire; ${ }^{2}$ Severn Hospice, Telford, Shropshire

\subsection{6/bmjspcare-2018-hospiceabs. 45}

Background Advance care planning (ACP) is a process of formal decision making and documentation for future reference. There's a risk that what is expressed during ACP may not be plausible within the available healthcare provision or be inappropriate. Deterioration of the patient's condition could mean that what was once possible is no longer. If this is not effectively communicated then there is potential for unnecessary distress, anguish and conflict. For this to be communicated effectively nuances such as the difference between; 'Choice', 'Preference' and 'Rights' are paramount. This understanding needs to be on both sides of the consultation table for effective communication.

Aim To explore the perceptions and understanding of the terms; 'Choice', Preference' and 'Rights' of the multidisciplinary hospice team in relation to aspects of ACP.

Methodology An electronic survey completed by clinical staff within Severn Hospice over a week period.

Results There were 32 respondents from eight different job roles. 69\% $(n=22)$ stated 'where you die' as a 'Preference' with $28 \%(n=9)$ stating it a 'Choice'. Explaining DNACPR demonstrated; 'Choice' 39\% ( $\mathrm{n}=12) /$ 'Preference' 29\% ( $\mathrm{n}=8) /$ 'Right' 35\% ( $n=11)$. Decisions regarding refusing care were mostly seen as a 'Right' $(62 \% \mathrm{n}=20)$ whereas requesting aspects of care such as admission and medications were perceived as 'Choice' $(40 \% \mathrm{n}=13)$ or 'Preference' $(37 \% \mathrm{n}=12)$. The qualitative aspect asked for perceived definitions of 'Choice', 'Preference' and 'Rights' which we split into multiple themes. 'Choice' had predominance of the themes of 'options' and 'information'. 'Preference' had predominant themes of 'options' and 'if possible'. Rights had a more 'legal' aspect.

Conclusion There is considerable heterogeneity in the understanding of the terms; 'Choice', Preference', and 'Rights', and even more so between the terms 'Choice' and 'Preference'. Given this is conducted upon hospice staff this gives measure for concern as potentially conflicting messages and understanding could be communicated.

\section{P-21 SPACE - A TOOL TO AID BETTER COMMUNICATION}

${ }^{1}$ Sioned Evans, ${ }^{2}$ Julie Theisinger. 'St Luke's Hospice, Plymouth, UK; ${ }^{2}$ St Luke's Hospital Team, Derriford Hospital, Plymouth, UK

\subsection{6/bmispcare-2018-hospiceabs.46}

Breaking significant news is difficult. This is compounded when space to have these conversations is limited. This was the issue raised by the Care Quality Commission at the last hospital inspection. With an interest in communication skills, I was asked as a member of the Hospital Palliative Care Team for some advice; what guidance should we follow to break significant news, so that even if we do find ourselves, literally in a tight corner, we can try and do our best.

The acronym SPACE was developed, space being the problem and in part, the solution.

Setting

Preparation

Ask, Active listening, Acknowledge feelings

Chunk and Check

Exit well

The letters represent principles of good communication that are based on well-researched, established models. The aim with SPACE is for it to be a simple, memorable acronym that will encourage and enable all in better communication. A successful outcome would be staff feeling more confident in breaking significant news and subsequently a better experience for patients and relatives. This model is currently being incorporated in to communication skills training in the hospital and in the hospice. We are also teaching bespoke groups. In conjunction with the hospice's graphic designer, SPACE cards have been developed that fit in to the ID badge 